\title{
PAPER \\ Performance Evaluation of MIMO-UWB Systems Using Measured Propagation Data and Proposal of Timing Control Scheme in LOS Environments
}

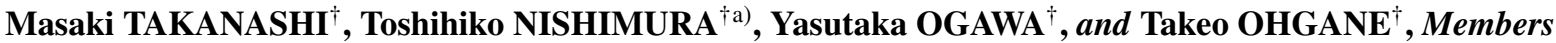

\begin{abstract}
SUMMARY Ultrawide-band impulse radio (UWB-IR) technology and multiple-input multiple-output (MIMO) systems have attracted interest regarding their use in next-generation high-speed radio communication. We have studied the use of MIMO ultrawide-band (MIMO-UWB) systems to enable higher-speed radio communication. We used frequency-domain equalization based on the minimum mean square error criterion (MMSEFDE) to reduce intersymbol interference (ISI) and co-channel interference (CCI) in MIMO-UWB systems. Because UWB systems are expected to be used for short-range wireless communication, MIMO-UWB systems will usually operate in line-of-sight (LOS) environments and direct waves will be received at the receiver side. Direct waves have high power and cause high correlations between antennas in such environments. Thus, it is thought that direct waves will adversely affect the performance of spatial filtering and equalization techniques used to enhance signal detection. To examine the feasibility of MIMO-UWB systems, we conducted MIMOUWB system propagation measurements in LOS environments. From the measurements, we found that the arrival time of direct waves from different transmitting antennas depends on the MIMO configuration. Because we can obtain high power from the direct waves, direct wave reception is critical for maximizing transmission performance. In this paper, we present our measurement results, and propose a way to improve performance using a method of transmit (Tx) and receive (Rx) timing control. We evaluate the bit error rate (BER) performance for this form of timing control using measured channel data.

key words: MIMO-UWB, LOS (line-of-sight) environment, frequencydomain equalization, transmit and receive timing control
\end{abstract}

\section{Introduction}

Ultrawide-band impulse radio (UWB-IR) technology [1], [2] and multiple-input multiple-output (MIMO) systems [3], [4] have attracted interest regarding their use in nextgeneration high-speed radio communication, and the use of MIMO ultrawide-band (MIMO-UWB) systems for higherspeed radio communication has been examined [5], [6]. In MIMO-UWB systems, intersymbol interference (ISI) usually occurs because of the wide-band transmission, and co-channel interference (CCI) occurs because independent signals are transmitted from multiple transmitting antennas. We have researched MIMO-UWB systems using the frequency domain equalization (FDE) technique [7] using the minimum mean square error (MMSE) spatial filtering (MMSE-FDE) technique. These studies were performed us-

Manuscript received September 29, 2008.

Manuscript revised February 24, 2009.

${ }^{\dagger}$ The authors are with the Graduate School of Information Science and Technology, Hokkaido University, Sapporo-shi, 0600814 Japan.

a) E-mail: nishim@ist.hokudai.ac.jp

DOI: 10.1587/transcom.E92.B.2698 ing computer simulations with multipath models such as the S-V model [8] or the IEEE 802.15.3a model [9]. However, these models are not necessarily applicable in MIMO environments because we must consider the fading correlation between antennas in such environments. UWB systems are expected to be used for short-range wireless communication; thus MIMO-UWB systems will usually operate in line-of-sight (LOS) environments, and direct waves will be received at the receiver side. (A LOS environment is assumed in the IEEE 802.15.3a model.) Direct waves have high power and cause high fading correlations between antennas. Thus, it has been conjectured that direct waves affect the performance of spatial filtering and equalization techniques used to enhance signal detection. Although wideband measurements have been reported in [10]-[13], these measurements only characterize single-input single-output (SISO)-UWB propagation phenomena. Yu et al. have reported MIMO-UWB propagation measurements, but these measurements are for a non-LOS (NLOS) environment [14].

We have measured the propagation of MIMO-UWB systems in LOS environments to investigate propagation phenomena and to examine the feasibility of MIMO-UWB systems. In this paper, we first show that the measured arrival time of direct waves from different transmitting antennas depends on the MIMO configuration, and that controlling the sampling instant for direct waves is critical to performance. To improve performance, we propose a transmit (Tx) and receive (Rx) timing control method that provides transmit and receive delays to enhance the reception of direct waves. We also show the effectiveness of this form of timing control in terms of bit error rate (BER) using measured channel data.

In Sect. 2 of this paper, we describe a MIMO-UWB system based on the MMSE-FDE technique. In Sect. 3, we present the results of measurements made in MIMO-UWB environments. We propose a Tx and Rx timing control scheme to improve the transmission performance in Sect. 4. In Sect. 5, we discuss our evaluation through computer simulations of the transmission performance using this timing control scheme in terms of BER. We conclude in Sect. 6.

\section{MIMO-UWB System Based on the MMSE-FDE Technique}

Here, we describe the transmission and reception system 


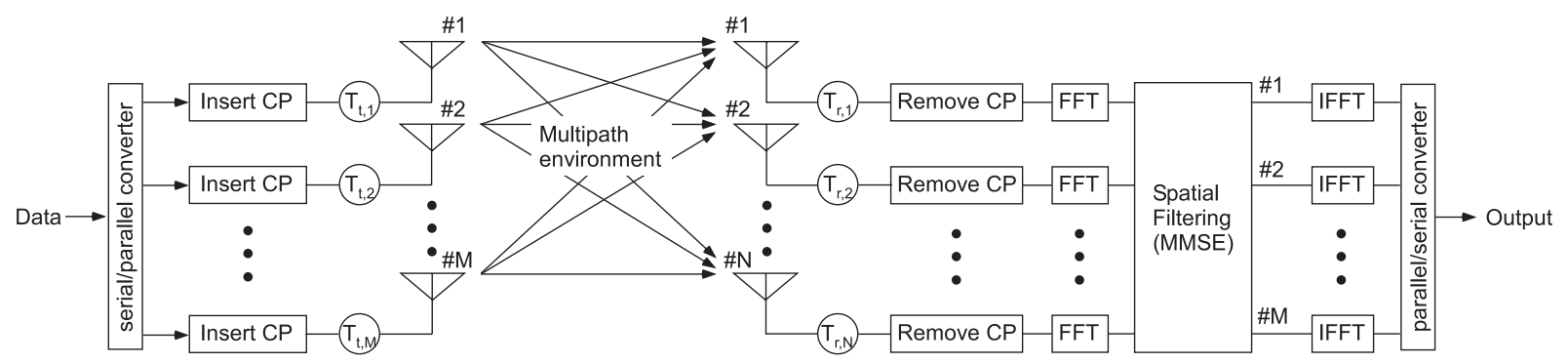

Fig. 1 Transmit and receive system in a MIMO environment.

that we assume in this paper. We consider a MIMO-UWB system using an MMSE-FDE as shown in Fig. 1. In the MIMO environment, we prepare $M$ transmit antennas and $N$ receive antennas. $T_{t, m}$ and $T_{r, n}$ denote delay devices for transmitting and receiving, respectively. These are needed for the Tx and Rx timing control, as we will later explain. In the UWB-IR system, when we transmit a pulse $p(t)$ from the $m$ th antenna, the received signal $r_{m, n}(t)$ at the $n$th antenna is expressed as

$$
r_{m, n}(t)=\sum_{j=0}^{\infty} \beta_{m, n}^{(j)} p\left(t-\tau_{m, n}^{(j)}\right),
$$

in multipath environments. In (1), $\beta_{m, n}^{(j)}$ and $\tau_{m, n}^{(j)}$ are the amplitude and arrival time of the $j$ th multipath signal between the $m$ th Tx antenna and the $n$th Rx antenna, respectively. For the FDE, before transmitting signals, we insert cyclic prefix (CP) symbols into the head of the data block. Then, when $K$ BPSK data symbols $\left(s_{k, m}= \pm 1\right)$ and $P$ CP symbols are transmitted, the received signal $x_{n}(t)$ is expressed as

$$
x_{n}(t)=\sum_{m=1}^{M} \sum_{k=-P}^{K-1} s_{k, m} r_{m, n}(t-k T)+z_{n}(t)
$$

where $s_{k, m}, T$ and $z_{n}(t)$ respectively denote the $k$ th transmitted symbol from the $m$ th Tx antenna, the transmit symbol interval and the noise at the $n$th $\mathrm{Rx}$ antenna.

In this paper, we assume that the multipath delay is less than $P$ symbols. At the receiver side, we first remove the $\mathrm{CP}$ symbols, and apply FFT to the received signal with an FFT window size of $K$. We can then obtain a frequency domain signal at the $n$th $\mathrm{Rx}$ antenna as follows:

$$
\begin{aligned}
X_{n}(f) & =\frac{1}{\sqrt{K}} \sum_{k=0}^{K-1} x_{n}(k T) \exp \left(-j 2 \pi \frac{f k}{K}\right) \\
& =\sum_{m=1}^{M} H_{m, n}(f) S_{m}(f)+Z_{n}(f),
\end{aligned}
$$

where $H_{m, n}(f)$ denotes the frequency domain channel from the $m$ th Tx antenna to the $n$th Rx antenna. $S_{m}(f)$ and $Z_{n}(f)$ respectively denote a transmit signal from the $m$ th Tx antenna and the noise at the $n$th Rx antenna in the frequency domain. These are expressed as follows:

$$
H_{m, n}(f)=\frac{1}{\sqrt{K}} \sum_{k=0}^{K-1} r_{m, n}(k T) \exp \left(-j 2 \pi \frac{f k}{K}\right)
$$

$$
\begin{aligned}
& S_{m}(f)=\frac{1}{\sqrt{K}} \sum_{k=0}^{K-1} s_{k, m} \exp \left(-j 2 \pi \frac{f k}{K}\right) \\
& Z_{n}(f)=\frac{1}{\sqrt{K}} \sum_{k=0}^{K-1} z_{n}(k T) \exp \left(-j 2 \pi \frac{f k}{K}\right) .
\end{aligned}
$$

We then define the frequency domain receive signal vector as

$$
\begin{aligned}
\boldsymbol{X}(f) & =\left[X_{1}(f) X_{2}(f) \ldots X_{N}(f)\right]^{T} \\
& =\sum_{m=1}^{M} S_{m}(f) \boldsymbol{H}_{m}(f)+\boldsymbol{Z}(f) .
\end{aligned}
$$

$\boldsymbol{H}_{m}(f)$ is the channel vector from the $m$ th Tx antenna and $\boldsymbol{Z}(f)$ is the noise vector:

$$
\begin{aligned}
& \boldsymbol{H}_{m}(f)=\left[\begin{array}{llll}
H_{m, 1}(f) & H_{m, 2}(f) & \ldots & H_{m, N}(f)
\end{array}\right]^{T} \\
& \boldsymbol{Z}(f)=\left[\begin{array}{llll}
Z_{1}(f) & Z_{2}(f) & \ldots & Z_{N}(f)
\end{array}\right]^{T} .
\end{aligned}
$$

The received signals involve not only ISI but also the CCI inherent in MIMO systems. We therefore need to cancel the two types of interference (ISI and CCI) simultaneously. We introduce weight vectors

$$
\boldsymbol{W}_{m}(f)=\left[\begin{array}{llll}
W_{m, 1}(f) & W_{m, 2}(f) \ldots W_{m, N}(f)
\end{array}\right]^{T} .
$$

If $\boldsymbol{W}_{m}(f)(m=1,2, \ldots, M)$ satisfy

$$
\boldsymbol{W}_{i}^{H}(f) \boldsymbol{H}_{j}(f)=\left\{\begin{array}{ll}
1 & (i=j) \\
0 & (i \neq j)
\end{array},\right.
$$

then we can cancel ISI and CCI. In this study, we obtain weight vectors $\boldsymbol{W}_{m}(f)$ satisfying the MMSE criterion as follows:

$$
\boldsymbol{W}_{m}(f)=\boldsymbol{R}^{-1}(f) \boldsymbol{H}_{m}(f) .
$$

Here, $\boldsymbol{R}(f)$ represents the correlation matrix given by

$$
\boldsymbol{R}(f)=\sum_{m=1}^{M} \boldsymbol{H}_{m}(f) \boldsymbol{H}_{m}^{H}(f)+\sigma^{2} \boldsymbol{I},
$$

where $\boldsymbol{I}$ is an $N \times N$ unit matrix. Using the weight vectors $\boldsymbol{W}_{m}(f)$, we obtain $K$ frequency domain outputs for the $m$ th Tx antenna:

$$
\begin{aligned}
\hat{S}_{m}(f) & =\boldsymbol{W}_{m}^{H}(f) \boldsymbol{X}(f) \\
& =S_{m}(f)+Z_{m}^{\prime}(f) .
\end{aligned}
$$


$Z_{m}^{\prime}(f)$ denotes the noise and residual interference in the frequency domain. Applying IFFT to $\hat{S}_{m}(f)$, we can then obtain $\hat{s}_{k, m}$, from which most of the ISI and CCI are removed:

$$
\begin{aligned}
\hat{s}_{k, m} & =\frac{1}{\sqrt{K}} \sum_{f=0}^{K-1} \hat{S}_{m}(f) \exp \left(j 2 \pi \frac{f k}{K}\right) \\
& =s_{k, m}+n_{k, m},
\end{aligned}
$$

where $n_{k, m}$ denotes the noise and residual interference.

\section{Wideband Propagation Measurement}

In this section, we describe our wideband propagation measurement. In the measurement, we obtained MIMO propagation data in a frequency domain using a vector network analyzer. We used broadband printed dipole antennas employing a self-complementary radiating element and a microstrip line feed [15] as Tx and Rx antennas, respectively. We show the measurement environment in Figs. 2 and 3. The antenna separation was $0.08 \mathrm{~m}$ at both the Tx end and the Rx end. The larger the antenna separation, the better the performance tends to be because the correlation is lower and the effect of mutual coupling is smaller. However, since a large antenna separation is not practical when mounting antennas on terminals for UWB communications, we chose $0.08 \mathrm{~m}$ as a reasonable value. As shown in Fig. 3, the antennas were mounted on an automated measurement system. This system was controlled by a laptop computer, which made it easy to change the antenna position. We measured the transmission coefficients for all Tx and Rx antenna pairs from $0.05 \mathrm{GHz}$ to $12.05 \mathrm{GHz}$ with a frequency interval of $0.0075 \mathrm{GHz}$; thus, the interval of time domain data that we could cover was $133.3 \mathrm{~ns}$. The length of the measurement site was about $5 \mathrm{~m}$, corresponding to a 16.7 -ns delay. The time interval of $133.3 \mathrm{~ns}$ can contain 8 reflections; thus, the frequency interval we used was valid for this measurement. We used RF switches to select each channel. The measurement system using the network analyzer and RF switches is shown in Fig. 4. We normalized the measured channel responses to the calibration data, which were obtained when the cables to the antenna ports from the RF switches were connected directly.

We examined three types of MIMO configuration, as shown in Fig. 5, and obtained 2100 items of data by changing the position of the Tx and Rx antenna array pair along the $\mathrm{x}$-axis and $\mathrm{y}$-axis for each configuration. This was performed because we needed a variety of channel data to evaluate the average BER performance. We moved the antenna system by $0.01 \mathrm{~m}$ increments along both the $\mathrm{x}$-axis and $\mathrm{y}$ axis. The Tx and Rx arrays were moved in parallel and fixed $1 \mathrm{~m}$ apart each time. The movement ranges along the $\mathrm{x}$-axis and $\mathrm{y}$-axis were $0.7 \mathrm{~m}$ and $0.3 \mathrm{~m}$, respectively. Because there were no objects between the Tx and Rx antennas, the direct wave arrived at each Rx antenna; that is, the setup was in a LOS environment. Because the measurement was performed in a shielded room, there was no interference from other radio systems. There were also no moving

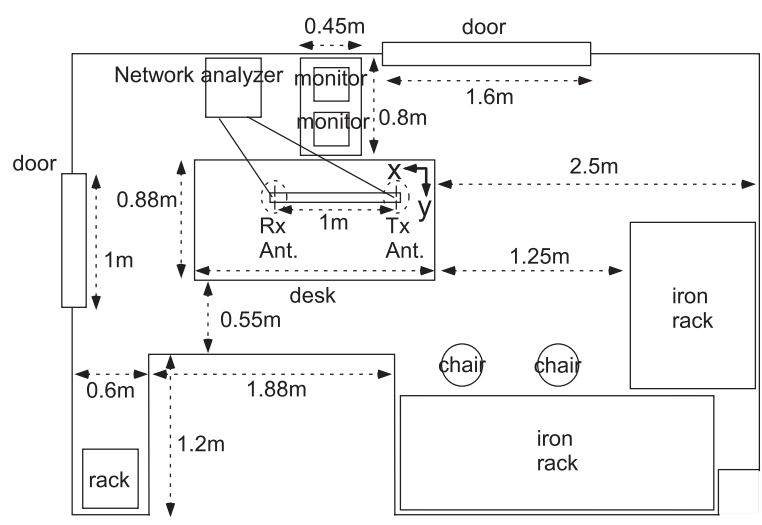

Fig. 2 Measurement environment (top view).

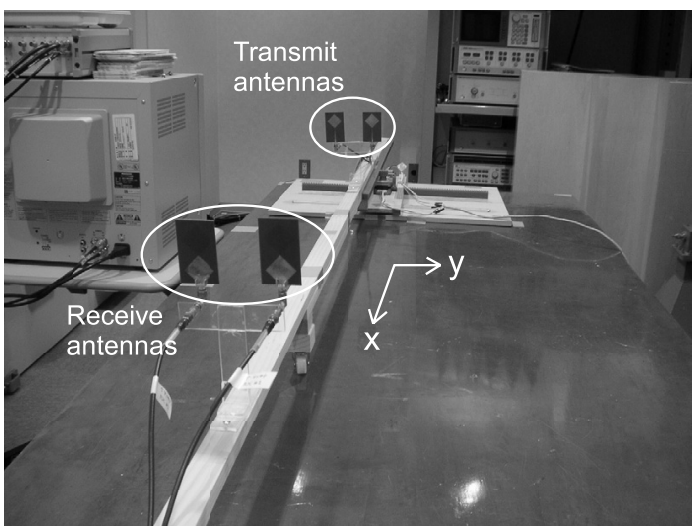

Fig. 3 MIMO antennas and measurement environment.

objects, which could have affected the propagation environment. In Fig. 6, we show an example of the measured data (solid curve) and the theoretical propagation loss (broken curve) for a distance of $1 \mathrm{~m}$. The propagation loss in a free space is given by

$$
L d B=-20 \log _{10}\left(\frac{4 \pi d}{\lambda}\right),
$$

where $d$ and $\lambda$ respectively denote the distance between the $\mathrm{Tx}$ and $\mathrm{Rx}$ antennas and the wavelength for each frequency.

As shown, the measured data decreased in agreement with the theoretical curve in the higher-frequency region. However, the channel response in the lower-frequency region was also low. This was due to the antenna property because this antenna was designed to meet the regulations for the UWB spectrum from 3.1 to $10.6 \mathrm{GHz}$.

In this study, we assumed a Tx monocycle pulse given by

$$
p(t)=\left[1-4 \pi(t / \tau)^{2}\right] \exp \left[-2 \pi(t / \tau)^{2}\right],
$$

where $\tau=0.2877$ ns. Figures 7-9 respectively show examples of received pulses for the MIMO configurations "pos 1," "pos2" and "pos3" shown in Fig. 5. These were calculated using the measured transmission data and the Tx monocycle pulse as follows. The monocycle pulse given by (21) 


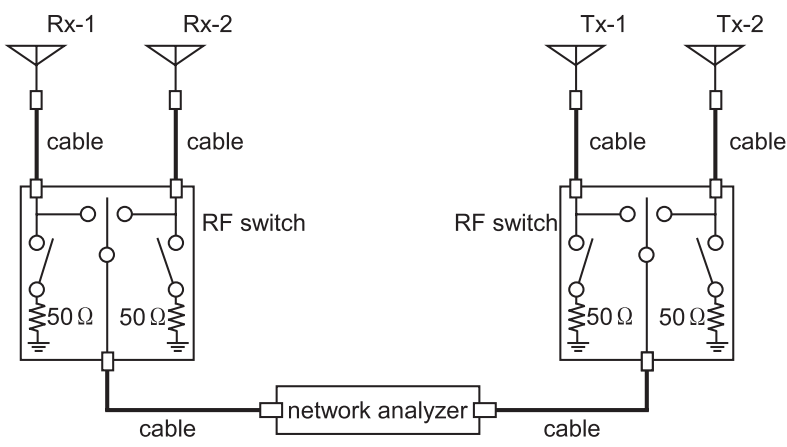

Fig. 4 Measurement system.

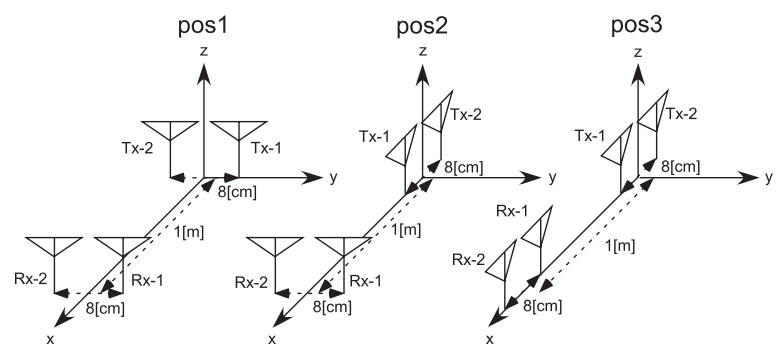

Fig. 5 MIMO configurations.

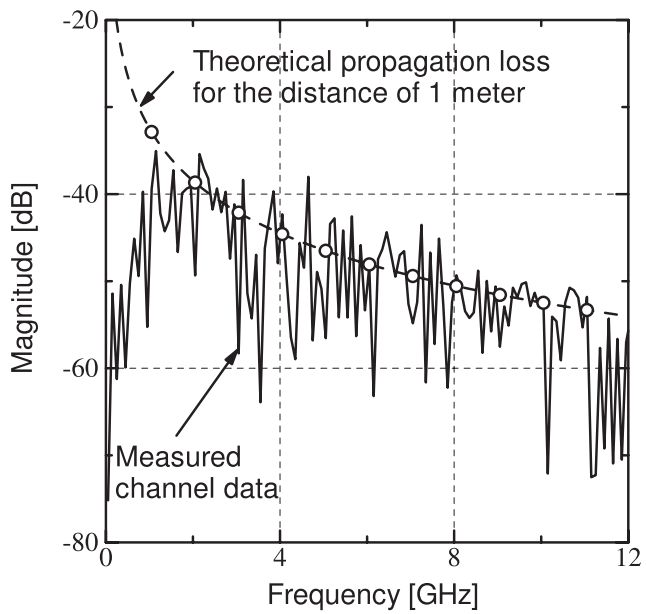

Fig. 6 Example of measured data.

was Fourier-transformed, and we obtained the frequency domain monocycle data. We then multiplied the measured frequency characteristics of the channel by the above frequency domain monocycle data. Finally, we obtained the time domain response by applying an inverse Fourier transform to the frequency domain data. For simplicity, no matched filter was employed in this case. We observed multiple reflections in the antenna, and multiple components were radiated from the antenna. Thus, the direct wave, which was not reflected by surrounding objects, consisted of multiple components. These components can be seen in the figures because the wide-band system had high resolution within the time domain. The same reflection phenomenon in the antenna has been described elsewhere [15]. It was caused by waves ra-
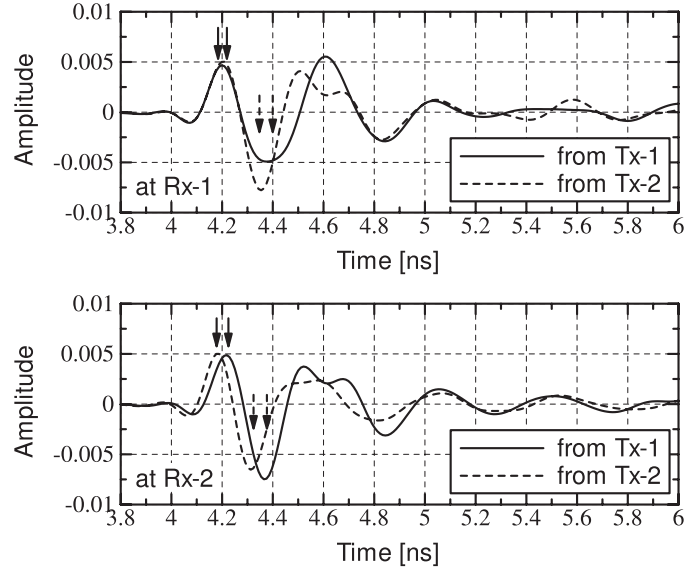

Fig. 7 Received pulse for "pos1."
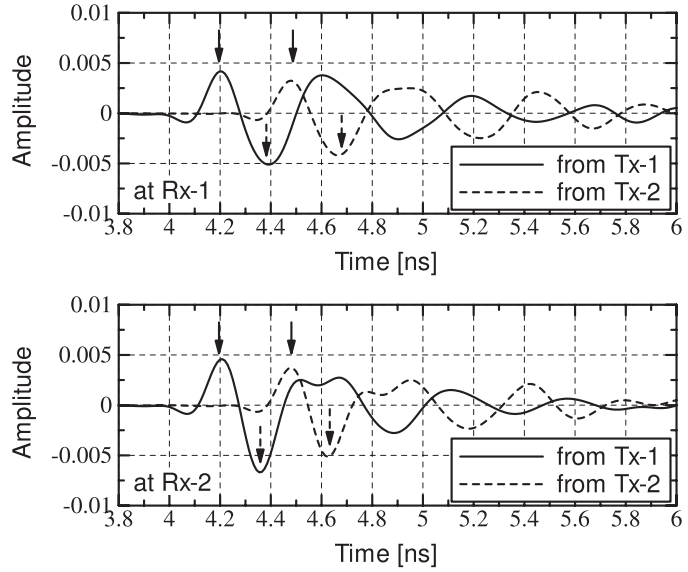

Fig. 8 Received pulse for "pos2."
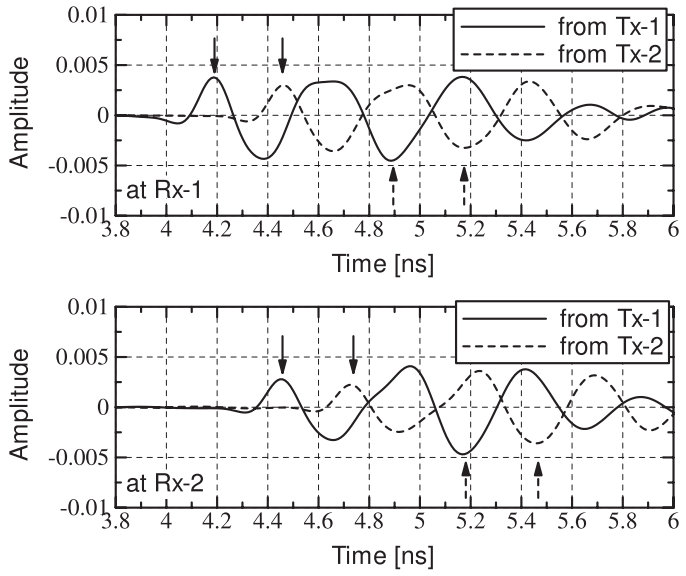

Fig. 9 Received pulse for "pos3."

diated from discontinuous points in a finite-length antenna. The same phenomenon has been observed in a monopole antenna for a narrow-band system by applying a superresolution algorithm [16]. In a $2 \times 2$ MIMO system, we have four direct waves. We indicate the fastest component in each direct wave by a solid arrow in the figures. The fastest 


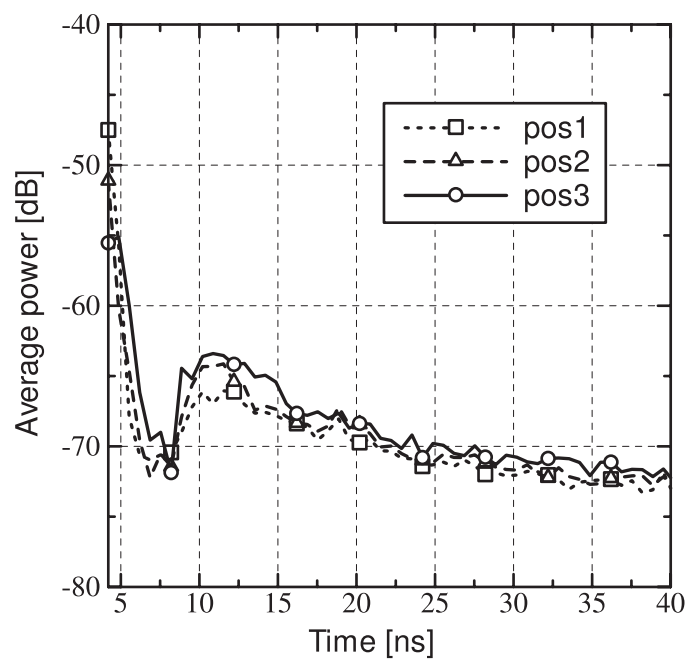

Fig. 10 Average power decay.

component denotes the peak whose delay is the shortest in each direct wave. We also indicate the largest component in each direct wave by a broken arrow. The largest component denotes the highest peak in each direct wave. These large components are used for timing control, as will later be explained. We next discuss the fastest direct wave components.

In Fig.7, we can see that all the fastest direct wave components arrived at the Rx antennas at about $4.2 \mathrm{~ns}$ for pos1. In Fig.8, we can see that although the fastest components from the first Tx antenna arrived at the Rx antennas at about $4.2 \mathrm{~ns}$, the fastest components from the second Tx antenna arrived at the Rx antennas at about $4.5 \mathrm{~ns}$ for pos2. This was because the second Tx antenna was $0.08 \mathrm{~m}$ farther from the Rx antenna system than the first Tx antenna. In Fig.9, we can see that the fastest component from each Tx antenna arrived at the Rx antennas at different times. According to these results, the arrival time of the direct waves differs for the various MIMO configurations because of the difference in propagation distance. We can then relate the difference in the arrival time of direct wave components to the difference in the MIMO configuration. This is important for MIMOUWB systems, as we will later explain.

In Fig. 10, we show the average power decay for each MIMO configuration. We can see that the average received power was higher and differed for the MIMO configurations in the shorter-delay region and that it was almost the same in the longer-delay region. In Figs. 11 and 12, we show the correlations at the Rx and Tx sides for each MIMO configuration. The correlations were calculated as

$$
\begin{aligned}
\rho_{n_{1}, n_{2}}(\tau) & =\frac{1}{\gamma_{n_{1}, n_{2}}(\tau)} \sum_{d=1}^{D} \sum_{m=1}^{M} r_{m, n_{1}}^{(d)}(\tau) r_{m, n_{2}}^{(d)}(\tau), \\
\gamma_{n_{1}, n_{2}}(\tau) & =\sqrt{\sum_{d=1}^{D} \sum_{m=1}^{M}\left(r_{m, n_{1}}^{(d)}(\tau)\right)^{2}} \times
\end{aligned}
$$

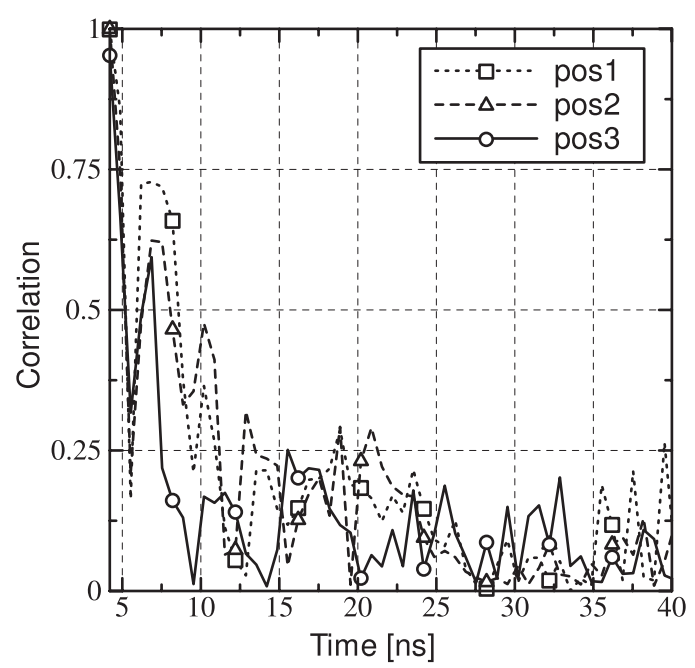

Fig. 11 Correlation between receive antennas.

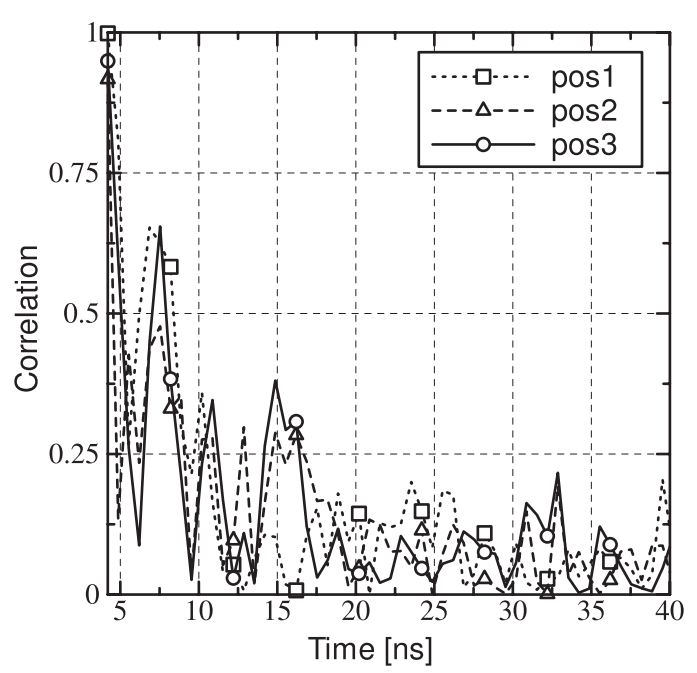

Fig. 12 Correlation between transmit antennas.

$$
\begin{gathered}
\sqrt{\sum_{d=1}^{D} \sum_{m=1}^{M}\left(r_{m, n_{2}}^{(d)}(\tau)\right)^{2}}, \\
\rho_{m_{1}, m_{2}}(\tau)=\frac{1}{\gamma_{m_{1}, m_{2}}(\tau)} \sum_{d=1}^{D} \sum_{n=1}^{N} r_{m_{1}, n}^{(d)}(\tau) r_{m_{2}, n}^{(d)}(\tau), \\
\gamma_{m_{1}, m_{2}}(\tau)= \\
\sqrt{\sum_{d=1}^{D} \sum_{n=1}^{N}\left(r_{m_{1}, n}^{(d)}(\tau)\right)^{2}} \times \\
\sqrt{\sum_{d=1}^{D} \sum_{n=1}^{N}\left(r_{m_{2}, n}^{(d)}(\tau)\right)^{2}} .
\end{gathered}
$$

$\rho_{n_{1}, n_{2}}(\tau)$ and $\rho_{m_{1}, m_{2}}(\tau)$ respectively denote the correlation between the $n_{1}$ th Rx antenna and the $n_{2}$ th Rx antenna at time $\tau$, and between the $m_{1}$ th Tx antenna and the $m_{2}$ th Tx antenna at time $\tau . \quad \gamma_{n_{1}, n_{2}}(\tau)$ and $\gamma_{m_{1}, m_{2}}(\tau)$ are respectively the normalization values for the $\mathrm{Rx}$ and $\mathrm{Tx}$ correlation values 
at time $\tau$. Moreover, $r_{m, n}^{(d)}(\tau)$ denotes the $d$ th measurement data from the $m$ th $\mathrm{Tx}$ antenna to the $n$th $\mathrm{Rx}$ antenna. Note that we obtained 2100 items of data by changing the antenna positions as stated. Thus, $D=2100$. From these results, we can see that the correlations are low in the longer-delay region, although in the shorter-delay region the correlations are very high. The high correlations are conjectured to be due to the direct waves. The differences in the direct wave arrival time are thought to lead to differences in correlation and transmission performance. Thus, differences in the arrival time of direct waves are likely to be critical for maximizing transmission performance, which will be discussed in the following sections.

\section{Transmit and Receive Timing Control}

In this section, we propose a $\mathrm{Tx}$ and $\mathrm{Rx}$ timing control scheme to improve performance. Here, we examine the receiving conditions for the MIMO configurations in detail. In Fig. 13, we schematically show the received direct waves in three cases when a single pulse is transmitted from two antennas (Tx-1, Tx-2). As stated in the previous section, the direct wave consists of multiple components. However, here it is illustrated as a single large pulse for simplicity in explaining the concept of the timing control.

We receive both direct waves at the first sampling time for "type1." This occurred in the case of pos1 due to the MIMO antenna configuration. For "type2," we receive only one direct wave at the first sampling time. This occurred in the case of pos2. On the other hand, for "type 3 ," one direct wave is received from the Tx-1 antenna by the Rx-1 antenna at the first sampling time, and another direct wave is received from the Tx-2 antenna by the Rx-2 antenna at the second sampling time. In each case, the arrival time of the direct wave is different. This can be realized not only by adjusting the MIMO antenna configuration but also by controlling the Tx timing and $\mathrm{Rx}$ timing. The Tx timing is controlled by adjusting the transmission instant, and the Rx timing is controlled by adjusting the sampling instant. These forms of control are realized using delay devices $\left(T_{t, m}\right.$ and
$\left.T_{r, n}\right)$, as shown in Fig. 1. Because we can control the reception of the direct waves through the timing control scheme, we can achieve better transmission performance, as will be shown in the next section.

Since the direct wave consists of multiple components, the timing control should be applied with respect to larger components to obtain higher received power. We used the components indicated by the broken arrows in Figs. 7-9.

\section{BER Evaluation}

In this section, we evaluate the BER performance using the

Table 1 Simulation parameters.

\begin{tabular}{c|c}
\hline Symbol (pulse) interval & $0.667 \mathrm{~ns}$ \\
\hline Sampling interval & $0.667 \mathrm{~ns}$ \\
\hline Modulation & BPSK \\
\hline Transmit and receive antennas & $(\mathrm{Tx}, \mathrm{Rx})=(2,2)$ \\
\hline Spatial filtering and equalization & MMSE-FDE \\
\hline Channel state information & known \\
\hline FFT window size $(\mathrm{K})$ & 1024 samples \\
\hline CP length & 256 samples \\
\hline
\end{tabular}

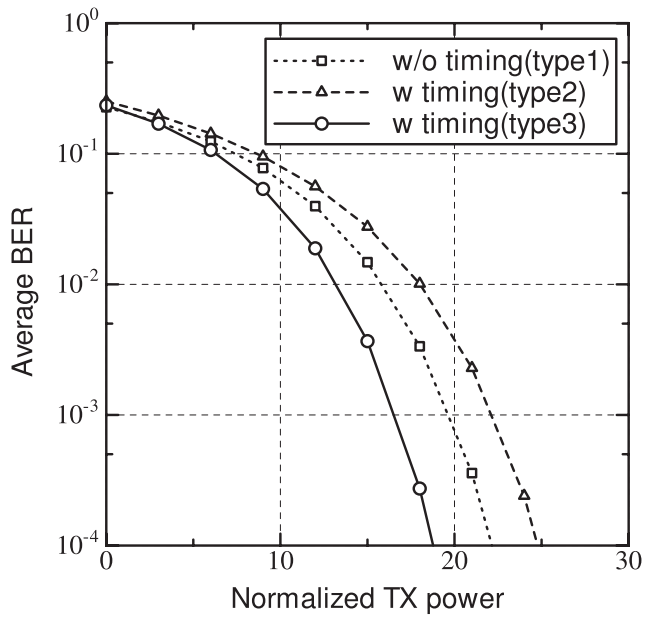

Fig. 14 BER performance for pos1 with timing control

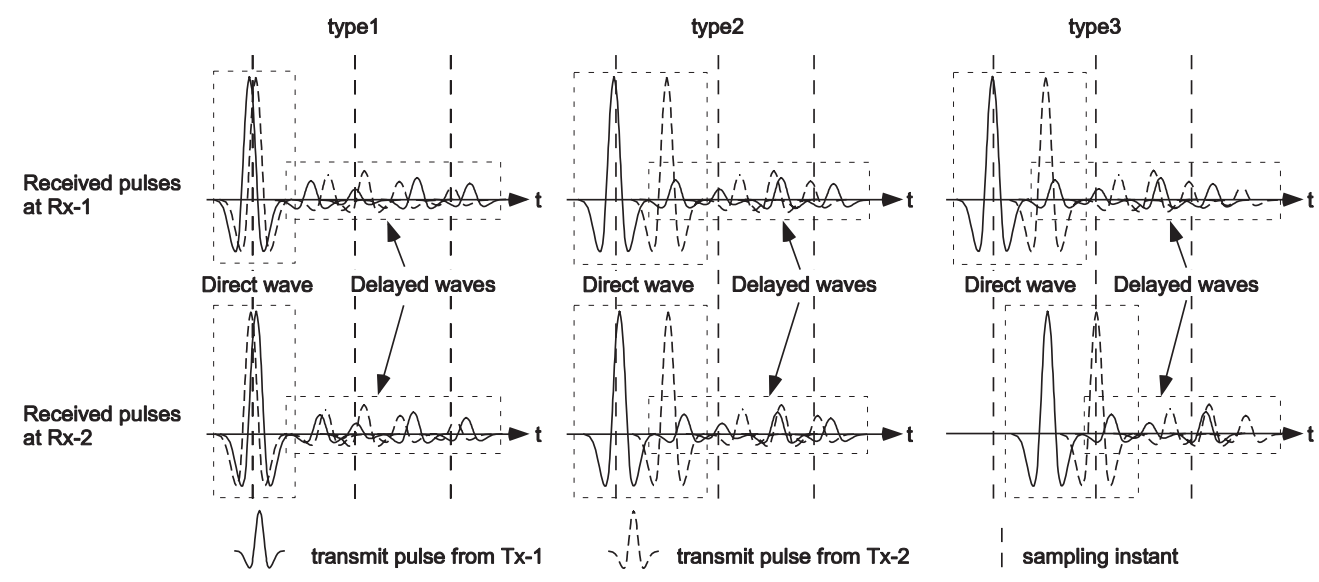

Fig. 13 Schematic figures of direct waves. 


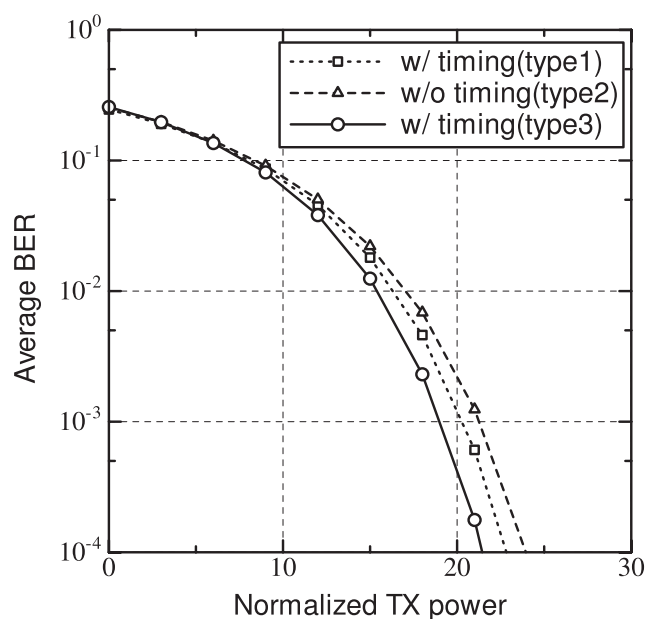

Fig. 15 BER performance for pos 2 with timing control.

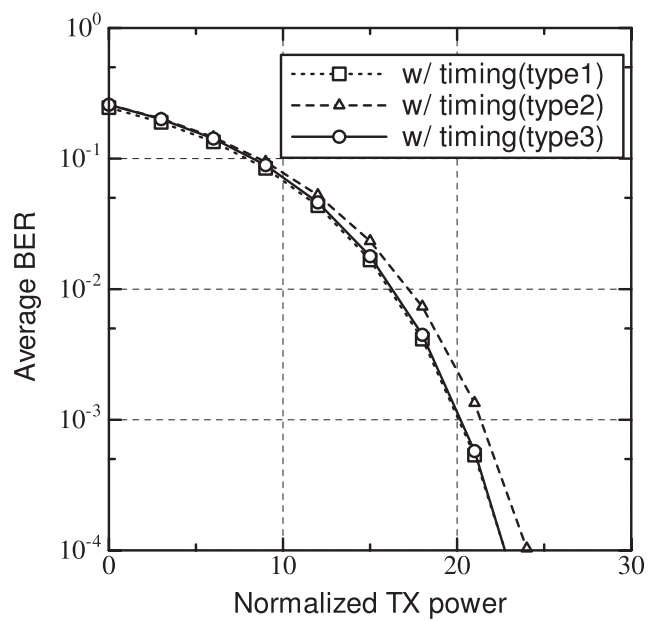

Fig. 16 BER performance for pos3 with timing control.

timing control scheme. We show the simulation parameters used for evaluating BER performance in Table 1. As stated, the components with larger amplitude are used for the timing control. In Figs. 14-16, we show BER performance versus normalized TX power. Here, the normalized TX power denotes the total transmit power normalized by the transmit power giving $E_{b} / N_{0}=0 \mathrm{~dB}$ when the signal is transmitted from a single transmit antenna. This transmit power was calculated using the theoretical equation of propagation loss for $1 \mathrm{~m}$ with the assumption that isotropic antennas at the transmitter and receiver are used in a free space, as assumed in (20). We indicate the transmission timing and sampling start time for the first pulse for each antenna position in Tables 2-4.

Figure 14 shows the BER performance for pos1. In this case, type 1 corresponds to not using the timing control; that is, type 1 is automatically realized with the pos 1 MIMO configuration without the timing control. From this figure, we can see that the type 3 timing control scheme enabled the greatest improvement in performance, while type 2 led to the worst performance.
Table 2 Transmit timing (Tx-1,Tx-2) and sampling start time ( $\mathrm{Rx}-1$, $\mathrm{Rx}-2$ ) for pos1.

\begin{tabular}{c|c|c|c}
\hline & type1 & type2 & type3 \\
\hline $\mathrm{Tx}-1$ & $0 \mathrm{~ns}$ & $0 \mathrm{~ns}$ & $0 \mathrm{~ns}$ \\
\hline $\mathrm{Tx}-2$ & $0 \mathrm{~ns}$ & $0.3 \mathrm{~ns}$ & $0.3 \mathrm{~ns}$ \\
\hline $\mathrm{Rx}-1$ & $4.34 \mathrm{~ns}$ & $4.34 \mathrm{~ns}$ & $4.34 \mathrm{~ns}$ \\
\hline $\mathrm{Rx}-2$ & $4.34 \mathrm{~ns}$ & $4.34 \mathrm{~ns}$ & $4.64 \mathrm{~ns}$ \\
\hline
\end{tabular}

Table 3 Transmit timing (Tx-1,Tx-2) and sampling start time (Rx-1, $\mathrm{Rx}-2)$ for $\operatorname{pos} 2$.

\begin{tabular}{c|c|c|c}
\hline & type 1 & type2 & type3 \\
\hline $\mathrm{Tx}-1$ & $0.26 \mathrm{~ns}$ & $0 \mathrm{~ns}$ & $0 \mathrm{~ns}$ \\
\hline $\mathrm{Tx}-2$ & $0 \mathrm{~ns}$ & $0 \mathrm{~ns}$ & $0 \mathrm{~ns}$ \\
\hline $\mathrm{Rx}-1$ & $4.65 \mathrm{~ns}$ & $4.39 \mathrm{~ns}$ & $4.39 \mathrm{~ns}$ \\
\hline $\mathrm{Rx}-2$ & $4.65 \mathrm{~ns}$ & $4.39 \mathrm{~ns}$ & $4.65 \mathrm{~ns}$ \\
\hline
\end{tabular}

Table 4 Transmit timing (Tx-1,Tx-2) and sampling start time (Rx-1, $\mathrm{Rx}-2)$ for pos3.

\begin{tabular}{c|c|c|c}
\hline & type1 & type2 & type3 \\
\hline $\mathrm{Tx}-1$ & $0.28 \mathrm{~ns}$ & $0 \mathrm{~ns}$ & $0 \mathrm{~ns}$ \\
\hline $\mathrm{Tx}-2$ & $0 \mathrm{~ns}$ & $0 \mathrm{~ns}$ & $0 \mathrm{~ns}$ \\
\hline $\mathrm{Rx}-1$ & $5.18 \mathrm{~ns}$ & $4.9 \mathrm{~ns}$ & $4.9 \mathrm{~ns}$ \\
\hline $\mathrm{Rx}-2$ & $5.46 \mathrm{~ns}$ & $5.18 \mathrm{~ns}$ & $5.46 \mathrm{~ns}$ \\
\hline
\end{tabular}

Figure 15 shows the BER performance for pos2. In this case, type 2 corresponds to not using the timing control. With the MIMO configuration, type 2 is automatically realized. Type 3 again performed best and type 2 performed worst.

Figure 16 shows the BER performance for pos3. The BER performance for type 1 was almost the same as that for type3, and that for type2 was the worst. These results indicate that type 3 is the best timing control scheme. We consider that the correlation is the lowest for type 3 , as will be shown below, and that the co-channel interference suppression is greatest in this case.

The correlation between antennas is critical to signal separation performance in MIMO systems. In this study, because we applied the MMSE-FDE technique for MIMO signal separation, we can consider the correlation value averaged over all the frequency points when analyzing the MIMO signal separation performance. The Rx correlation value between the $n_{1}$ th and $n_{2}$ th $\mathrm{Rx}$ antennas, and the Tx correlation value between the $m_{1}$ th and $m_{2}$ th Tx antennas are expressed as

$$
\begin{aligned}
& \rho_{n_{1}, n_{2}}=\frac{1}{\gamma_{n_{1}, n_{2}}} \sum_{d=1}^{D} \sum_{m=1}^{M} \sum_{f=0}^{K-1} H_{m, n_{1}}^{(d) *}(f) H_{m, n_{2}}^{(d)}(f), \\
& \gamma_{n_{1}, n_{2}}=\sqrt{\sum_{d=1}^{D} \sum_{m=1}^{M} \sum_{f=0}^{K-1}\left|H_{m, n_{1}}^{(d)}(f)\right|^{2}} \\
& \times \sqrt{\sum_{d=1}^{D} \sum_{m=1}^{M} \sum_{f=0}^{K-1}\left|H_{m, n_{2}}^{(d)}(f)\right|^{2}},
\end{aligned}
$$




$$
\begin{aligned}
\rho_{m_{1}, m_{2}}= & \frac{1}{\gamma_{m_{1}, m_{2}}} \sum_{d=1}^{D} \sum_{n=1}^{N} \sum_{f=0}^{K-1} H_{m_{1}, n}^{(d) *}(f) H_{m_{2}, n}^{(d)}(f), \\
\gamma_{m_{1}, m_{2}}= & \sqrt{\sum_{d=1}^{D} \sum_{n=1}^{N} \sum_{f=0}^{K-1}\left|H_{m_{1}, n}^{(d)}(f)\right|^{2}} \\
& \times \sqrt{\sum_{d=1}^{D} \sum_{n=1}^{N} \sum_{f=0}^{K-1}\left|H_{m_{2}, n}^{(d)}(f)\right|^{2}}
\end{aligned}
$$

where $H_{m, n}^{(d)}(f)$ denotes the frequency domain channel between the $m$ th Tx antenna and the $n$th $\mathrm{Rx}$ antenna for the $d$ th measurement data. $\gamma_{n_{1}, n_{2}}$ and $\gamma_{m_{1}, m_{2}}$ are the normalization values for the $\mathrm{Rx}$ and $\mathrm{Tx}$ correlation values, respectively. We also have $D=2100, M=N=2$ and $K=1024$. For cross-correlation functions, we have the Fourier transform theorem:

$$
\begin{aligned}
& R(\tau)=\int_{-\infty}^{\infty} v_{1}(t) v_{2}(t+\tau) d t \\
& R(\tau)=\int_{-\infty}^{\infty} V_{1}^{*}(f) V_{2}(f) \exp (j 2 \pi f \tau) d f,
\end{aligned}
$$

where $V_{1}(f)$ and $V_{2}(f)$ are Fourier transforms of the time domain functions $v_{1}(t)$ and $v_{2}(t)$, respectively. We apply the discrete Fourier transform at $\tau=0$ to obtain the relationship

$$
\sum_{f=0}^{K-1} V_{1}^{*}(f) V_{2}(f)=\sum_{k=0}^{K-1} v_{1}(k T) v_{2}(k T) .
$$

Using this relationship for the channel state information, we obtain the correlation equations from (26) and (28) as follows:

$$
\begin{aligned}
\rho_{n_{1}, n_{2}}= & \frac{1}{\gamma_{n_{1}, n_{2}}} \sum_{d=1}^{D} \sum_{m=1}^{M} \sum_{k=0}^{K-1} h_{m, n_{1}}^{(d)}(k T) h_{m, n_{2}}^{(d)}(k T) \\
\gamma_{n_{1}, n_{2}}= & \sqrt{\sum_{d=1}^{D} \sum_{m=1}^{M} \sum_{k=0}^{K-1}\left(h_{m, n_{1}}^{(d)}(k T)\right)^{2}} \\
& \times \sqrt{\sum_{d=1}^{D} \sum_{m=1}^{M} \sum_{k=0}^{K-1}\left(h_{m, n_{2}}^{(d)}(k T)\right)^{2}} \\
\rho_{m_{1}, m_{2}}= & \frac{1}{\gamma_{m_{1}, m_{2}}} \sum_{d=1}^{D} \sum_{n=1}^{N} \sum_{k=0}^{K-1} h_{m_{1}, n}^{(d)}(k T) h_{m_{2}, n}^{(d)}(k T), \\
\gamma_{m_{1}, m_{2}}= & \sqrt{\sum_{d=1}^{D} \sum_{n=1}^{N} \sum_{k=0}^{K-1}\left(h_{m_{1}, n}^{(d)}(k T)\right)^{2}} \\
& \times \sqrt{\sum_{d=1}^{D} \sum_{n=1}^{N} \sum_{k=0}^{K-1}\left(h_{m_{2}, n}^{(d)}(k T)\right)^{2}} \\
h_{m, n}^{(d)}(k T)= & r_{m, n}^{(d)}\left(k T-T_{t, m}-T_{r, n}\right) .
\end{aligned}
$$

Here, $h_{m, n}^{(d)}(k T)$ denotes the time domain channel between the $m$-th transmit antenna and the $n$-th receive antenna when the timing control scheme is employed for the $d$-th measurement data.
Table 5 Correlation and received power using the timing control schemes for pos 1 .

\begin{tabular}{c|c|c|c}
\hline & type1 & type2 & type3 \\
\hline Correlation(Tx) & 0.718 & 0.190 & 0.158 \\
\hline Correlation(Rx) & 0.729 & 0.613 & 0.222 \\
\hline Received power(Tx-1) & $0 \mathrm{~dB}$ & $0 \mathrm{~dB}$ & $-1.48 \mathrm{~dB}$ \\
\hline Received power(Tx-2) & $0.01 \mathrm{~dB}$ & $-3.8 \mathrm{~dB}$ & $-1.38 \mathrm{~dB}$ \\
\hline
\end{tabular}

Although we have already given correlations in (22) and (24), these correlations have been defined to analyze the propagation phenomena in MIMO-UWB environments, and they represent the correlations of the arrival waves for each time. The correlations in (33) and (35) are defined to analyze MIMO signal separation performance. In this section, "correlation" denotes the values averaged over the frequency domain, as given by (26) and (28) (or (33) and (35)). Therefore, we conjecture that when the correlation values are low, we can achieve better signal separation performance using the MMSE-FDE technique.

In Table 5, we show the correlation values between the antennas and the received power from the transmit antennas for the pos 1 configuration using the timing control schemes. The received power in Table 5 denotes the total power received by the two antennas from each Tx antenna. The received power in Table 5 is normalized by that from the first Tx antenna without the timing control scheme for the pos 1 configuration.

We can see that the received power was higher in the type 1 case than in the other cases. This was because we could obtain direct wave components from both of the Tx antennas. However, the correlation was also high. On the other hand, in the type 3 case, the received power was lower than in the type 1 case, but the correlation was also lower. We consider the better transmission performance of type 3 to have been due to the lower correlation. For type2, the correlation was not high, but the received power from the second Tx antenna was much lower than that from the first Tx antenna. Therefore, we consider that the transmission quality differed between the two streams and that the transmission performance was attributable to the worse stream of the two.

In Tables 6 and 7, we show the correlation values between the antennas and the received power from the Tx antennas for configurations pos 2 and pos 3 using the timing control schemes, respectively. Similarly to pos 1 , the received power shown in Tables 6 and 7 is normalized by that from the first $\mathrm{Tx}$ antenna without the timing control scheme in configurations pos 2 and pos 3 , respectively. From Table 6, we can see that the received power increased when the type 1 timing control was applied, and the correlation decreased when the type 3 timing control was applied for configuration pos2. Considering the BER performance, the type 3 timing control was the best scheme in this configuration.

According to Table 7 , the received power increased when the type1 timing control was applied for configuration pos3, and the correlation was low for the type 3 timing control. Using this configuration, the BER performances for 
Table 6 Correlation and received power using the timing control schemes for pos 2 .

\begin{tabular}{c|c|c|c}
\hline & type1 & type2 & type3 \\
\hline Correlation(Tx) & 0.581 & 0.008 & 0.141 \\
\hline Correlation(Rx) & 0.553 & 0.493 & 0.220 \\
\hline Received power(Tx-1) & $0 \mathrm{~dB}$ & $0 \mathrm{~dB}$ & $-1.29 \mathrm{~dB}$ \\
\hline Received power(Tx-2) & $-1.29 \mathrm{~dB}$ & $-2.74 \mathrm{~dB}$ & $-1.96, \mathrm{~dB}$ \\
\hline
\end{tabular}

Table 7 Correlation and received power using the timing control schemes for pos3.

\begin{tabular}{c|c|c|c}
\hline & type 1 & type 2 & type3 \\
\hline Correlation(Tx) & 0.41 & 0.218 & 0.165 \\
\hline Correlation(Rx) & 0.398 & 0.339 & 0.214 \\
\hline Received power(Tx-1) & $0.73 \mathrm{~dB}$ & $0.86 \mathrm{~dB}$ & $0 \mathrm{~dB}$ \\
\hline Received power(Tx-2) & $-0.91 \mathrm{~dB}$ & $-3.28 \mathrm{~dB}$ & $-2.5 \mathrm{~dB}$ \\
\hline
\end{tabular}

type 1 and type 3 were almost the same.

From (33) and (35), we can see that the correlation is a summation of multiplications of time domain channels for all the sampling points. Because the time domain channel corresponding to the direct wave has high power, the correlation is strongly affected by this channel. Through the type 3 timing control, we can reduce the multiplication values for the case when the direct wave is not received by the same antenna since the transmission timing is delayed by half of the pulse time. Although the received power is then decreased, we can reduce the correlations given by (33) and (35). We consider that the lower correlation improves the MIMO detection and transmission performances. As stated in Sect. 3, the direct wave consists of multiple components, and the waveform is not simple, as shown in Fig. 13. However, from Tables 5-7, we can see that by controlling the timing of the larger components, we can adjust the received power and correlations. These results also indicate that the type 3 timing control is the best scheme for enhancing transmission performance.

In LOS environments, direct waves increase the correlation between antennas. This degrades the performance of MIMO space division multiplexing. We examined the performance of the proposed $2 \times 2$ MIMO system and the 1 $\times 2$ SIMO space diversity system. We considered the cases of $2 \mathrm{bits} / \mathrm{symbol}(2 \mathrm{bps} / \mathrm{Hz}$ ) and $4 \mathrm{bits} / \mathrm{symbol}(4 \mathrm{bps} / \mathrm{Hz})$. In the 2 bits/symbol case, we examined a $2 \times 2$ MIMO system with BPSK and a $1 \times 2$ SIMO system with 2 bit pulse amplitude modulation (2-PAM). In the 4 bits/symbol case, we used a $2 \times 2$ MIMO system with 2 -PAM and a $1 \times 2$ SIMO system with 4 bit PAM (4-PAM). We used the type 3 transmission scheme for the MIMO system. Figure 17 shows the average BER in the above cases. In the 2 bits/symbol case, the $1 \times 2$ SIMO system performed better than the proposed MIMO system. However, in the 4 bits/symbol case, the BER performance of the proposed MIMO system was better than that of the SIMO system. From these results, we can conclude that the proposed MIMO scheme is effective when multilevel modulation is used to increase transmission rate. Although no detailed explanation is given in this paper, if we use a more sophisticated reception scheme, such as a

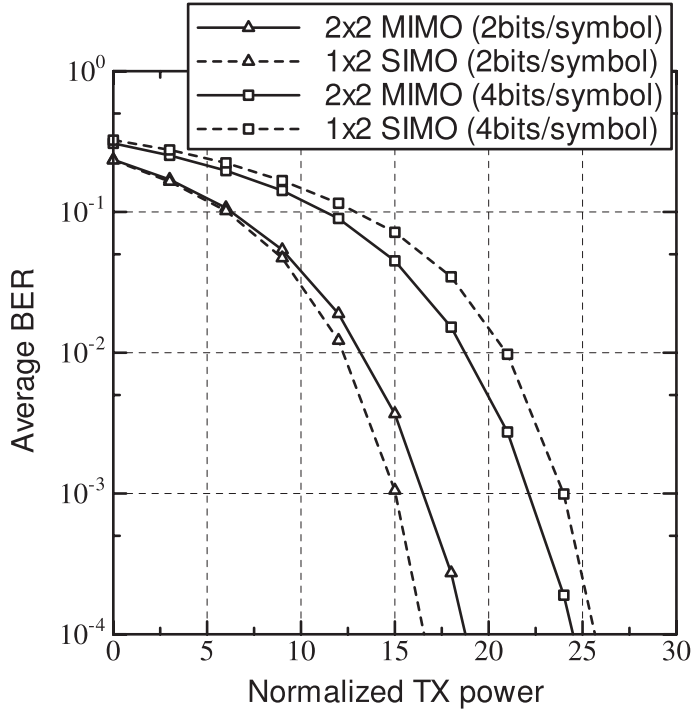

Fig. 17 BER performance for $2 \times 2$ MIMO system and $1 \times 2$ SIMO system.

parallel interference canceller, the $2 \times 2$ MIMO system outperforms the $1 \times 2$ SIMO system even in the 2 bits/symbol case.

\section{Conclusion}

We have presented MIMO-UWB measurement results and proposed a method of Tx and Rx timing control to improve performance. Through the measurements, we have shown that the arrival time of direct waves depends on the MIMO configuration but that the signal arrival behavior in the longer-delay region does not depend on the configuration. We proposed an optimum form of Tx and Rx timing control for direct waves. The analysis of BER performance and correlation demonstrated that the optimum timing control decreased the correlation and maximized the transmission performance. In this study, we assumed that the timing is known at the transmitter and receiver. However, we still need a timing estimation technique and a means of providing timing feedback to the transmitter; we will consider these in our future work.

\section{Acknowledgements}

We thank Dr. Michitaka Ameya and Dr. Manabu Yamamoto of Hokkaido University for providing the UWB antennas.

\section{References}

[1] M.Z. Win and R.A. Scholtz, "Ultra-wide bandwidth time-hopping spread-spectrum impulse radio for wireless multi-access communications," IEEE Trans. Commun., vol.48, no.4, pp.679-691, April 2000.

[2] S. Roy, J.R. Foerster, V.S. Somayazulu, and D.G. Leeper, "Ultrawideband radio design: The promise of high-speed, short-range wireless connectivity," Proc. IEEE, vol.92, no.2, pp.295-311, Feb. 2004

[3] G.J. Foschini and M.J. Gans, "On limits of wireless communications 
in a fading environment when using multiple antennas," Wirel. Pers. Commun., vol.6, no.3, pp.311-335, 1998.

[4] G.J. Foschini, G.D. Golden, R.A. Valenzuela, and P.W. Wolniansky, "Simplified processing for high spectral efficiency wireless communication employing multi-element arrays," IEEE J. Sel. Areas Commun., vol.17, no.11, pp.1841-1852, Nov. 1999.

[5] L. Zhiwei, B. Premkumar, and A.S. Madhukumar, "MMSE detection for high data rate UWB MIMO systems," Proc. IEEE Vehicular Technology Conference. IEEE VTC2004, vol.2, pp.1463-1467, Sept. 2004.

[6] W. Siriwongpairat, M. Olfat, and K.J.R. Liu, "On the performance evaluation of TH and DS UWB MIMO systems," WCNC2004, vol.3, pp.1800-1805, March 2004.

[7] D. Falconer, S.L. Ariyavisitakul, A.B. Seeyar, and B. Eidson, "Frequency domain equalization for single-carrier broadband wireless systems,” IEEE Commun. Mag., vol.40, pp.58-66, April 2002.

[8] A.A.M. Saleh and R.A. Valenzuela, "A statistical model for indoor multipath propagation,” IEEE J. Sel. Areas. Commun., vol.5, no.2, pp.128-137, Feb. 1987.

[9] J. Foerster, "P802.15-02/490r1-SG3a-Channel-ModelingSubcommittee-Report-Final.doc," http:/grouper.ieee.org /groups/ $802 / 15 /$ pub/

[10] R.J. Cramer, R.A. Scholtz, and M.Z. Win, "Evaluation of an ultra wide-band propagation channel," IEEE Trans. Antennas Propag., vol.50, no.5, pp.561-570, May 2002.

[11] D. Cassioli, M.Z. Win, and A.F. Molisch, "The ultra-wide bandwidth indoor channel: From statistical model to simulations," IEEE J. Sel. Areas Commun., vol.20, no.6, pp.1247-1257, Aug. 2002

[12] K. Haneda, J. Takada, and T. Kobayashi, "Double directional ultra wideband channel characterization in a line-of-sight home environment," IEICE Trans. Fundamentals, vol.E88-A, no.9, pp.2264 2271, Sept. 2005.

[13] Y. Suzuki and T. Kobayashi, "Ultra wideband signal propagation in desktop environments," IEICE Trans. Fundamentals, vol.E88-A, no.9, pp.2272-2278, Sept. 2005.

[14] K. Yu, M. Bengtsson, B. Ottersten, D. McNamara, P. Karlsson, and M. Beach, "A wideband statistical model for NLOS indoor MIMO channels," Proc. IEEE Vehicular Technology Conference. IEEE VTC2002, vol.1, pp.370-374, May 2002.

[15] M. Ameya, M. Yamamoto, T. Nojima, and K. Itoh, "Broadband printed dipole antenna employing self-complementary radiating element and microstrip line feed," IEICE Trans. Commun. (Japanese Edition), vol.J88-B, no.9, pp.1662-1673, Sept. 2005.

[16] Y. Kondo, K. Itoh, M. Ohmiya, and Y. Ogawa, "Time domain response of a monopole antenna," IEICE Technical Report, A.P88-25, June 1988.

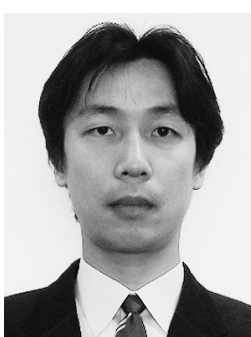

Toshihiko Nishimura received his B.S. and M.S. degrees in physics and a Ph.D. degree in electronics engineering from Hokkaido University, Sapporo, Japan, in 1992, 1994 and 1997 , respectively. In 1998, he joined the Graduate School of Engineering (since reorganized to the Graduate School of Information Science and Technology) at Hokkaido University, where he is currently an Assistant Professor of the Graduate School of Information Science and Technology. His current research interests are in MIMO systems using smart antenna techniques. Dr. Nishimura received the Young Researchers' Award of IEICE Japan in 2000 and the Best Paper Award from IEICE Japan in 2007. Dr. Nishimura is a member of the IEEE.

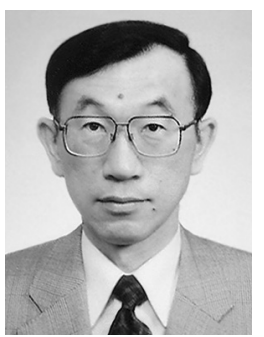

Yasutaka Ogawa received his B.E., M.E. and Ph.D. degrees from Hokkaido University, Sapporo, Japan, in 1973, 1975 and 1978, respectively. Since 1979, he has been with Hokkaido University, where he is currently a Professor of the Graduate School of Information Science and Technology. During 1992-1993, he was with ElectroScience Laboratory, the Ohio State University, U.S.A., as a Visiting Scholar, on leave from Hokkaido University. His interests are in adaptive antennas, mobile communications, superresolution techniques and MIMO systems. Dr. Ogawa received the Yasujiro Niwa Outstanding Paper Award in 1978, the Young Researchers' Award of IEICE Japan in 1982 and the Best Paper Award from IEICE Japan in 2007. Dr. Ogawa is a senior member of the IEEE.

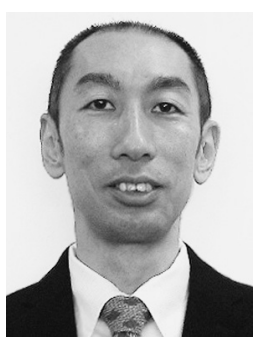

Takeo Ohgane received his B.E., M.E. and $\mathrm{Ph} . \mathrm{D}$. degrees in electronics engineering from Hokkaido University, Sapporo, Japan, in 1984 1986 and 1994, respectively. From 1986 to 1992, he was with Communications Research Laboratory, Ministry of Posts and Telecommunications. From 1992 to 1995, he was on assignment at ATR Optical and Radio Communications Research Laboratory. Since 1995, he has been with Hokkaido University, where he is an Associate Professor. During 2005-2006, he was at the Centre for Communications Research, University of Bristol, U.K., as a Visiting Fellow. His interests are in MIMO signal processing for wireless communications. Dr. Ohgane received the IEEE AP-S Tokyo Chapter Young Engineer Award in 1993, the Young Researchers' Award of IEICE Japan in 1990 and the Best Paper Award from IEICE Japan in 2007. Dr. Ohgane is a member of the IEEE.

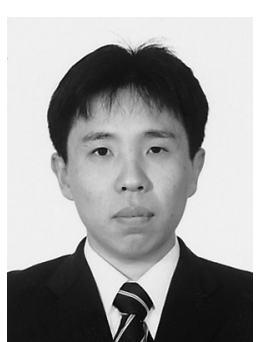

Masaki Takanashi received his B.E., M.E. and Ph.D. degrees from Hokkaido University, Sapporo, Japan, in 2001, 2003 and 2007, respectively. He is currently with Toyota Central R\&D Labs., Inc. His research interests are in superresolution techniques, ultrawide-band systems and signal processing for wireless communications. 